\title{
Nanorobots as sensors for cancer microenvironment
}

Debayan Dasgupta ${ }^{1}$, Dharma Pally2, Deepak K. Saini2,3, Ramray Bhat ${ }^{2}$ and Ambarish Ghosh ${ }^{1,4}$

Centre for Nano Science and Engineering, Indian Institute of Science, Bangalore 560012, India, ${ }^{2}$ Department of Molecular Reproduction, Development and Genetics, Indian Institute of Science, Bangalore 560012, India, ${ }^{3}$ Centre for Biosystems Science and Engineering, IISc, Bangalore 560012, India, ${ }^{4}$ Department of Physics, Indian Institute of Science, Bangalore 560012, India

Key ideas:

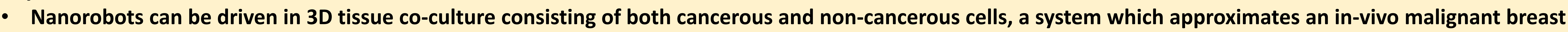
tumor.

- Nanorobots can sense physico-chemical changes in cancer-sculpted extracellular matrix (ECM).

\section{Experimental Setup}

Nanorobots move due to rotating magnetic field generated by triaxial Helmholtz coil.

Nanorobots are injected into tumor-like environment and moved for 30 minutes along a random direction.
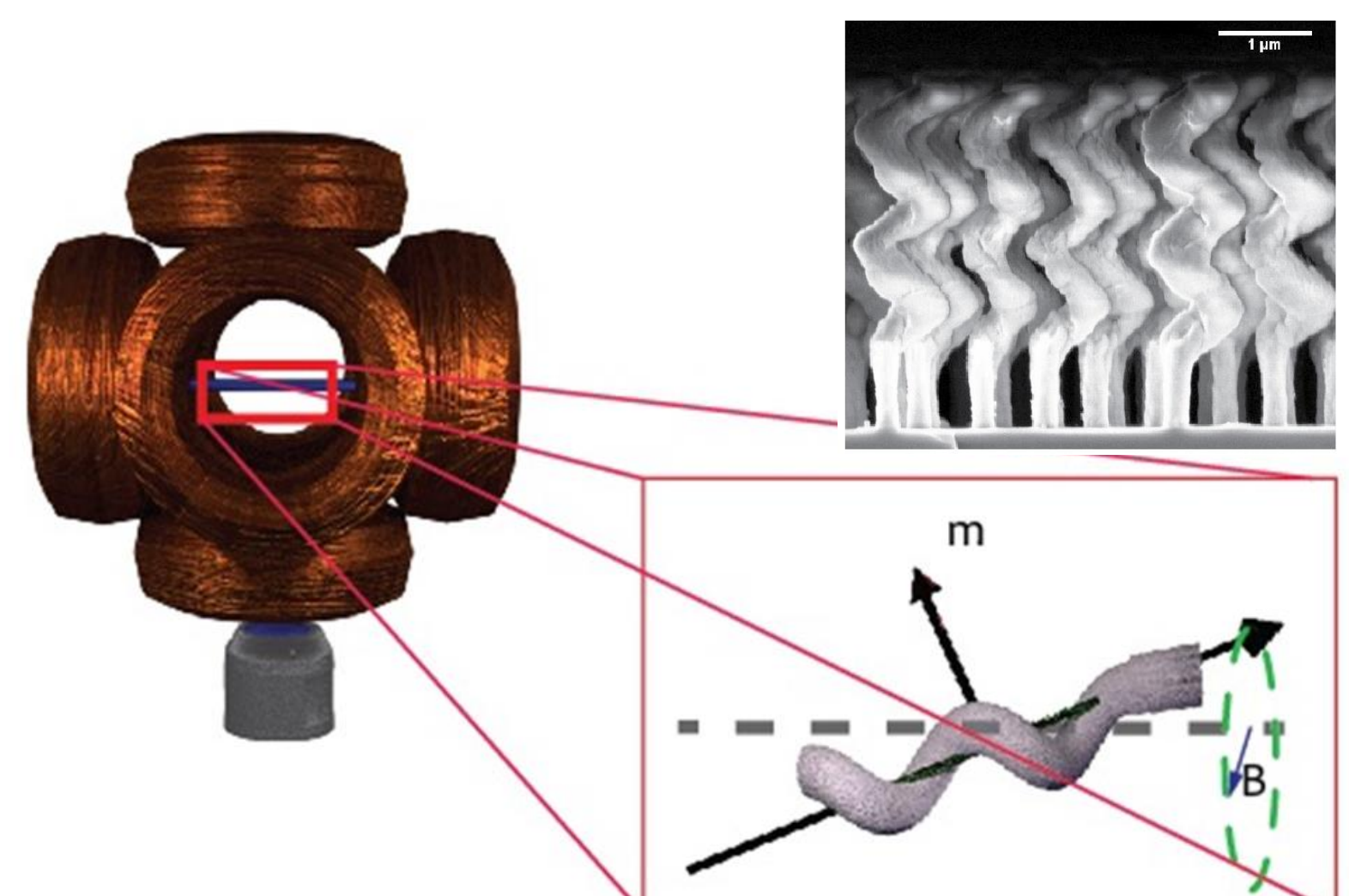

Nanorobots adhere to the cancer ECM but not near non-cancerous ECM

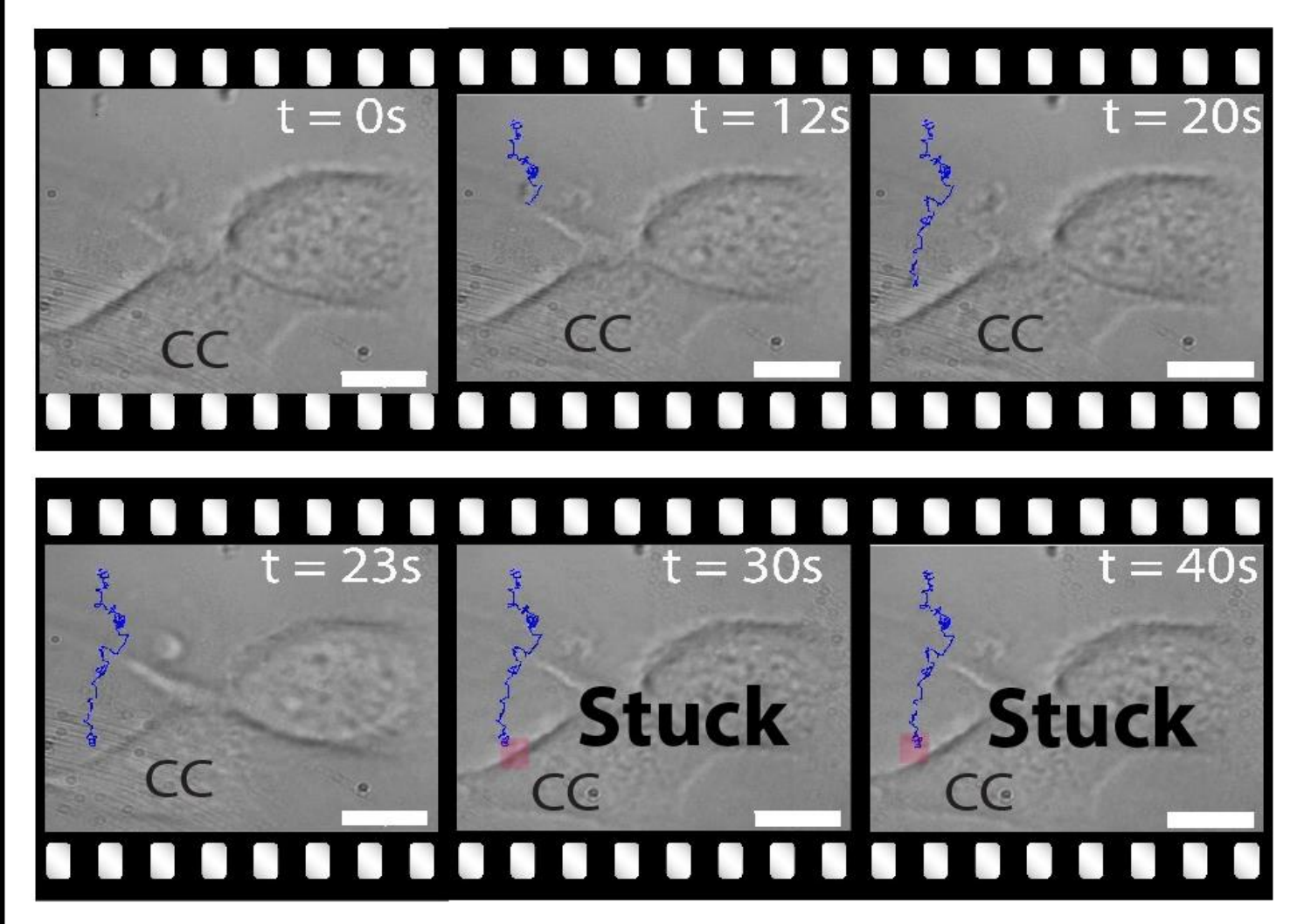

Trajectory of nanorobot near a cancer cell:

Nanorobot gets adhered to the ECM in the vicinity of cancer cell$$
\text { 列 }
$$

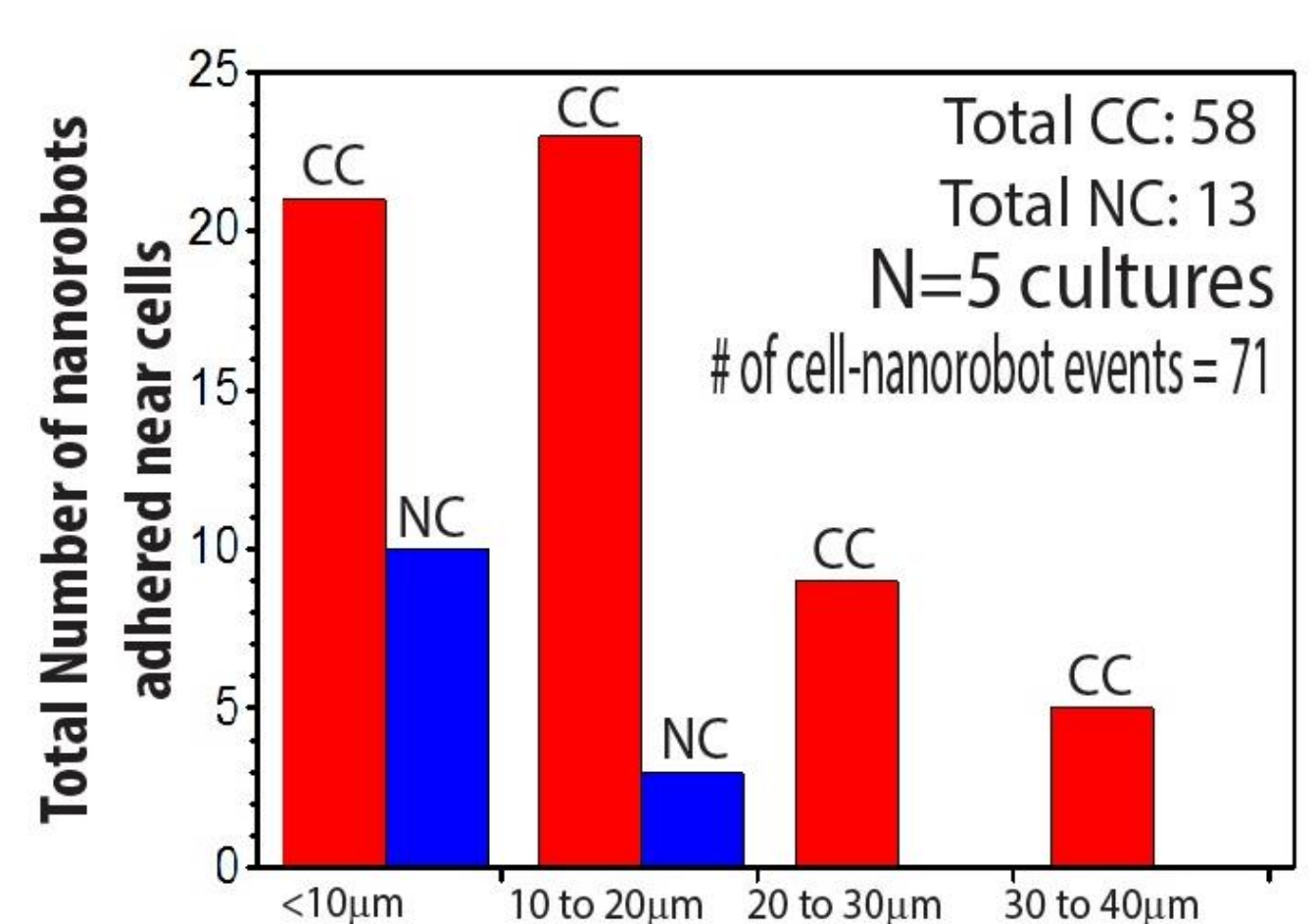

Distance from the cell membrane

\section{Adhesion force is correlated to metastatic}

potential

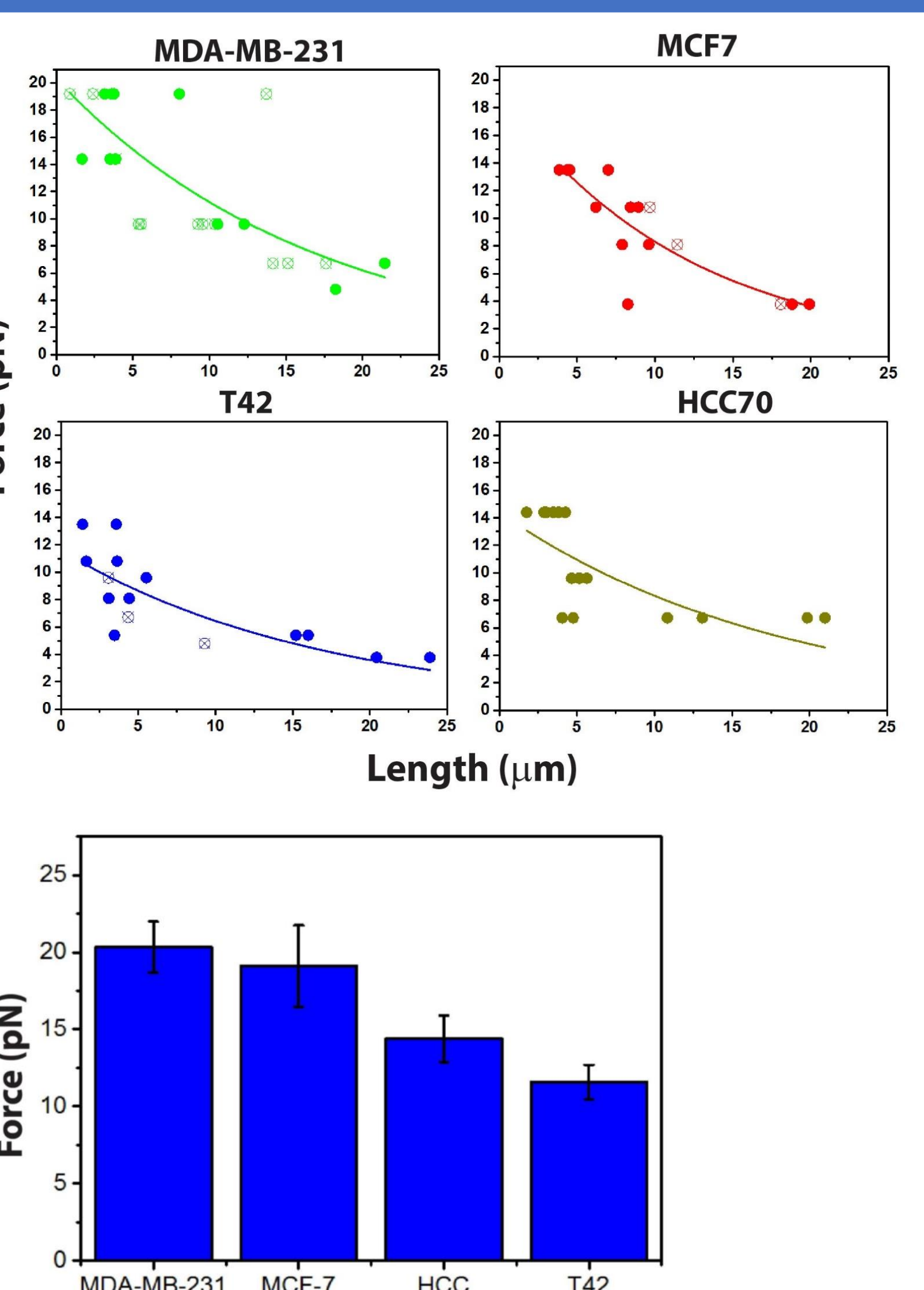
them.

- Estimated $F_{0}$ for various cell lines. potential.

a2,3-linked sialic acid may be the primary cause of adhesion

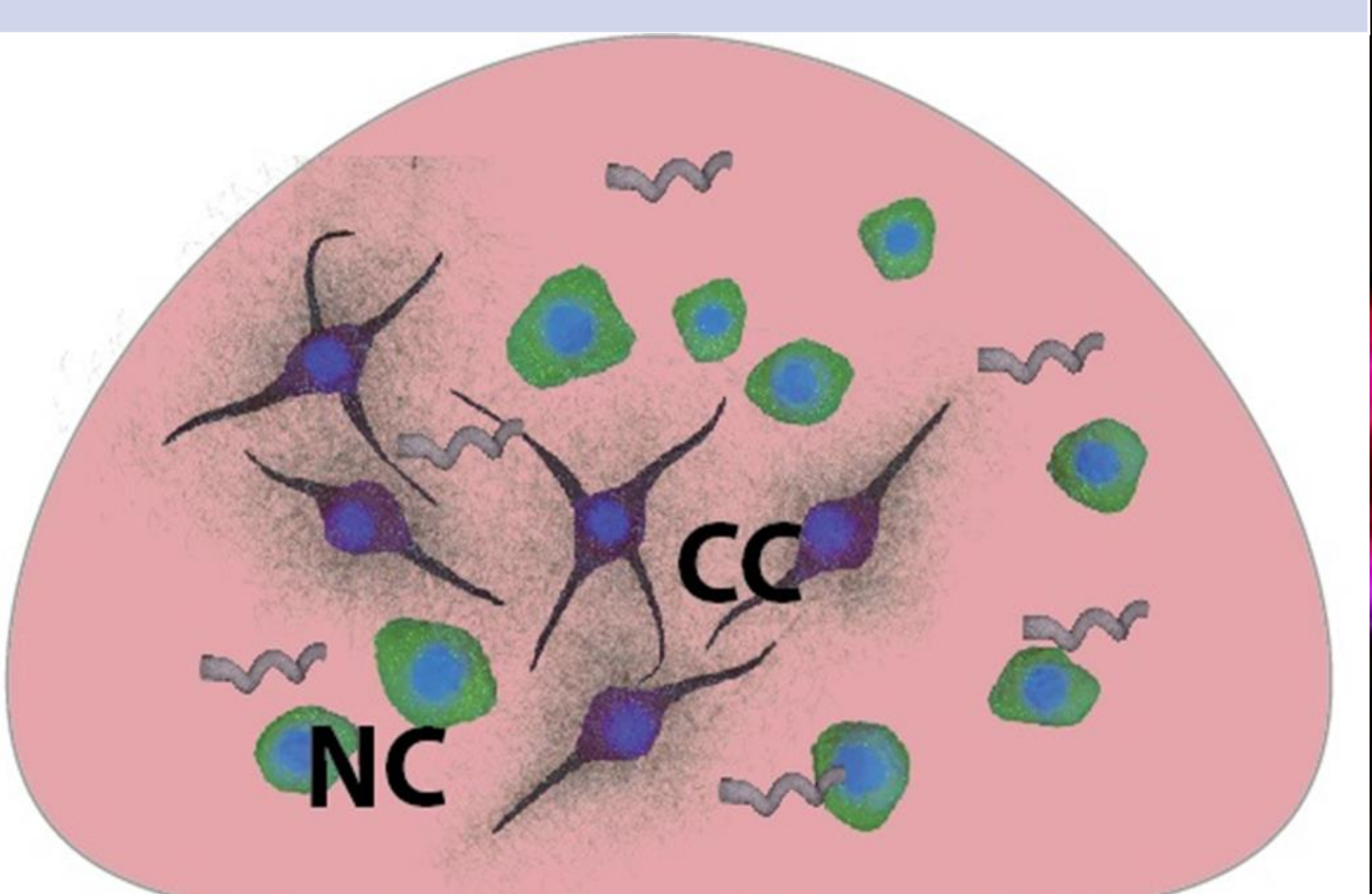

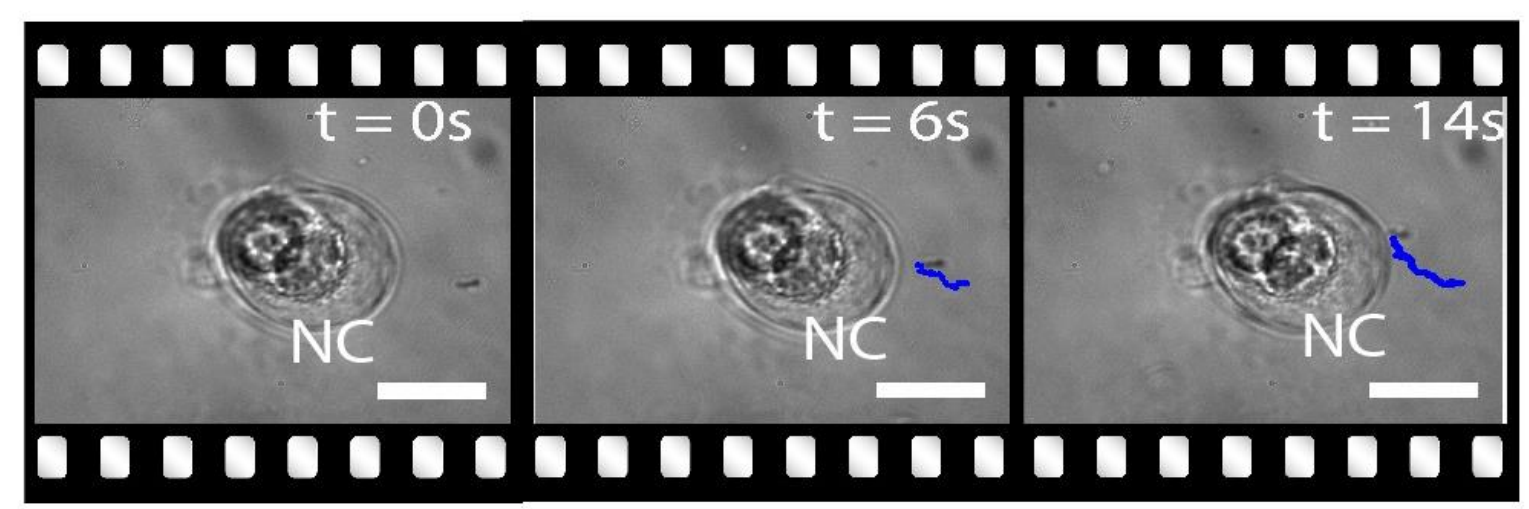

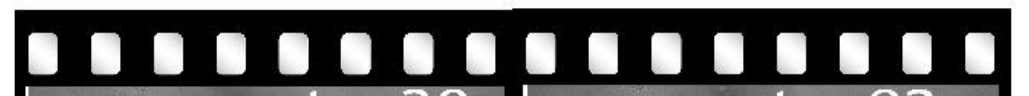
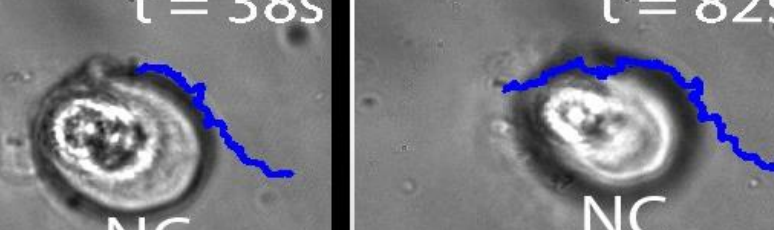

\section{0}

Trajectory of nanorobot near a normal cell: Nanorobot can be maneuvered near a normal cell

- A total of 376 cells were sampled, of which 203 cells were cancer cells and 173 were normal cells.

- Total 71 cells were found to have adhered nanorobots in their vicinity, of which 58 were cancer cells and 13 were normal cells. 2,3-sialic acid

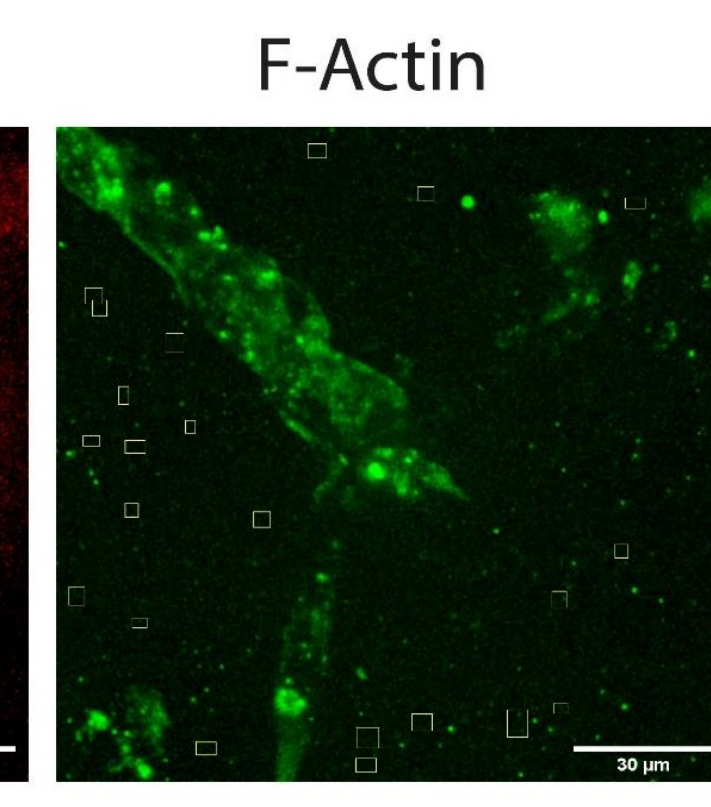

- Adhered nanorobots are spatially co-located with sialylated cancer ECM.

- The extent of sialic acid spread was measured to be $\sim 40 \mu \mathrm{m}$

Negatively charged PFO coated nanorobots do not get adhered

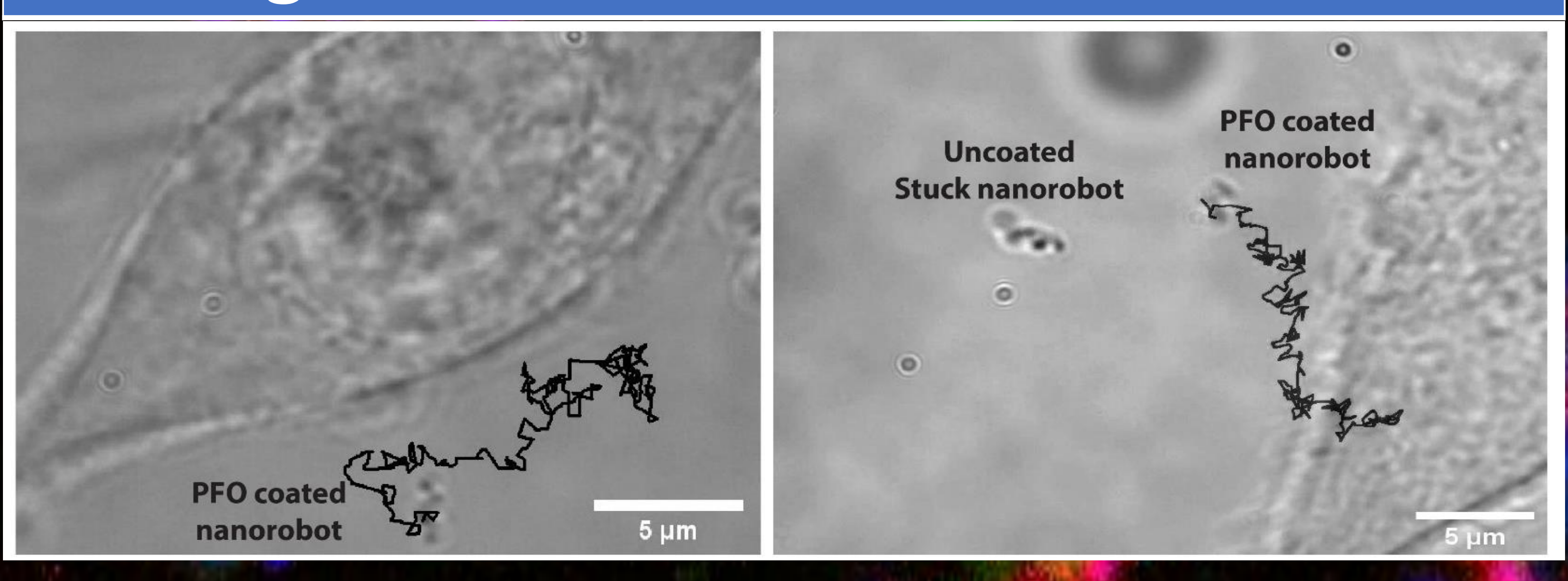

Anisotropy of nanorobot adhesion is correlated to ECM sialylation

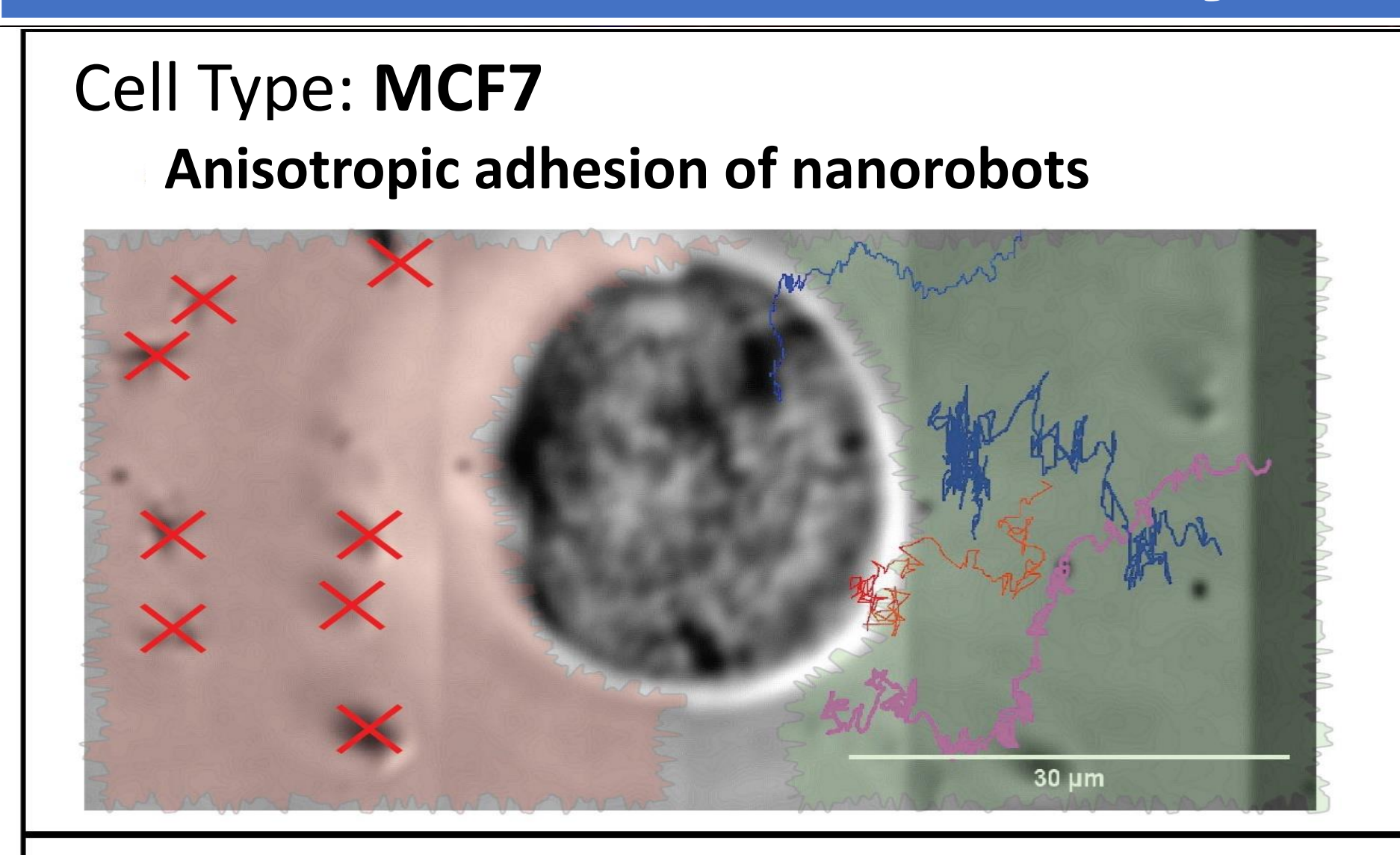

Cell Type: MDA-MB-231

Isotropic adhesion of nanorobots

Anisotropic distribution of Sialic acid

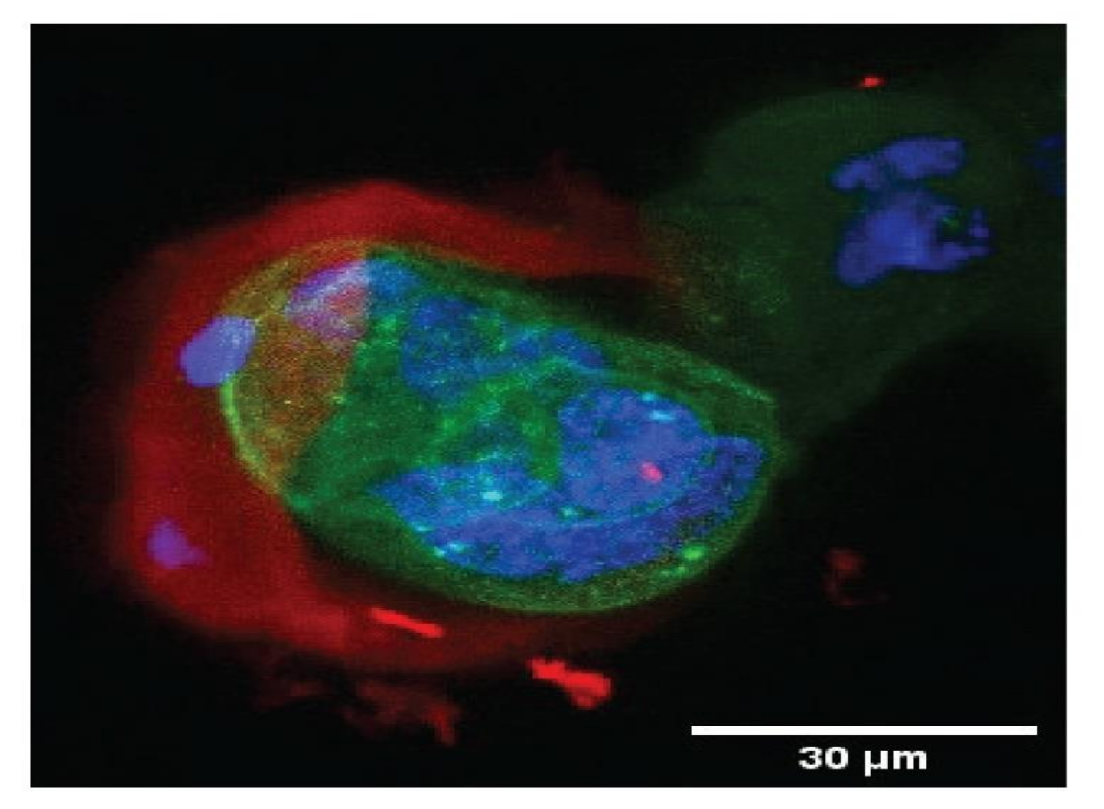

Isotropic distribution of Sialic acid
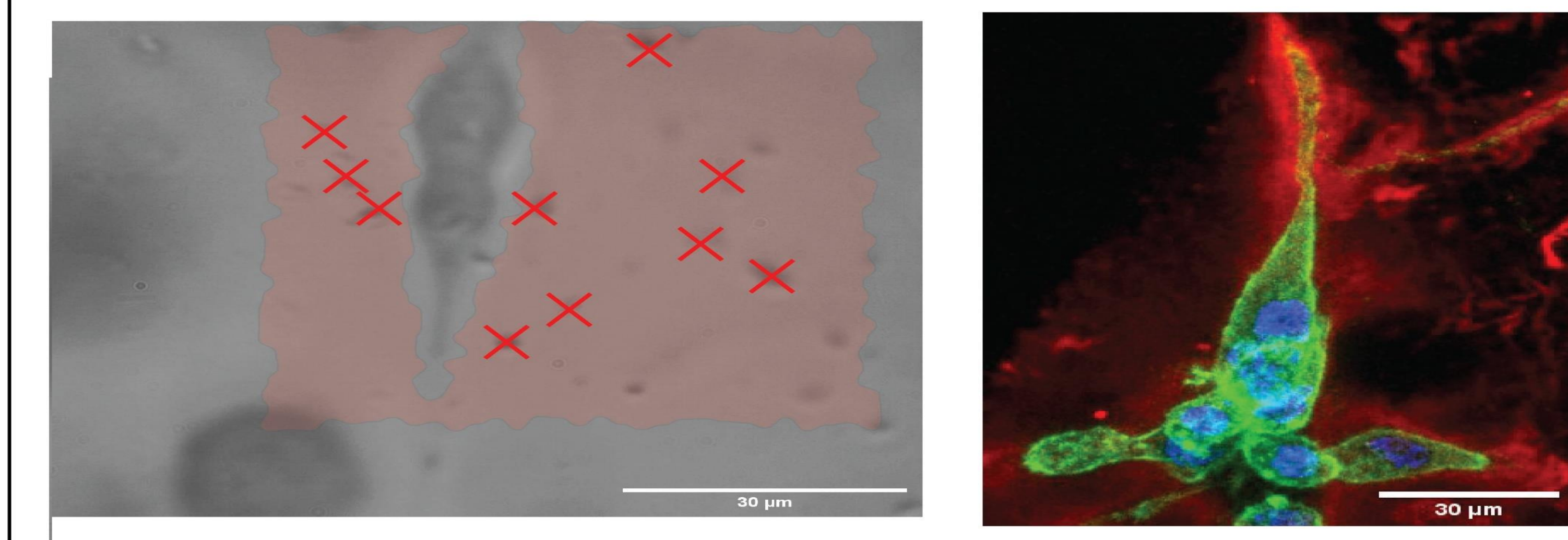

Physical effects of invading cancer cells are manifest up to a length of $\sim 40 \mu \mathrm{m}$ from the cell surface within the ECM surrounding

- $F_{0}$ increases for cells with higher metastatic
Acknowledgement. This research was supported in part by DBT and DST. DDG and DP will like to thank IISC and the Ministry of Fellowship (SRF). RB would like to acknowledge support from the SERE ECR Grant (Human Resource Development (MHRD), Government of India for providing the Senior Research Grant, Wellcome Trust-DBT India Alliance Fellowship (WELTO041) and the DBT-I partnership program (BT/PR27952/INF/22/212/2018)

\section{Summary}

- Dynamics of nanorobot $\rightarrow$ spatially differentiate cancerous and noncancerous cell microenvironment.

Nanorobot adhesion near cancer cells $\rightarrow$ different charge conditions of the cancer sculpted ECM.

- Physical effects of invading cancer cell $\rightarrow \sim 40 \mu \mathrm{m}$ from the cell surface.

Specific sialic acid linkages within the ECM secreted by the cells

- Implications for future in-vivo cancer targeting.

Patent: Charge-based methods for studying a biochemical micro-environment, Debayan Dasgupta, Dharma Patent: he Tejashwar Reddy Pally, Deepak Saini, Ramray Bhat, Ambarish Ghosh, Indian patent (provisional) application, 